Letters to the Editors

from page 247

don't help the visual effect. It is obviously not in the patient's best interest.

2. BFUs have better results than SFUs from either a quantity or quality point of view.

3. In Dr. Beehner's report, both of his patients' data show 12-14 month yield rates less than 5.5-7 months without any exception. If the result is reversed, then it is much easier to explain. Now how can we find a reason for it? We always believe that the transplanted hair will grow for a long time. What would be the result at 18 and 24 months? A follow-up study would

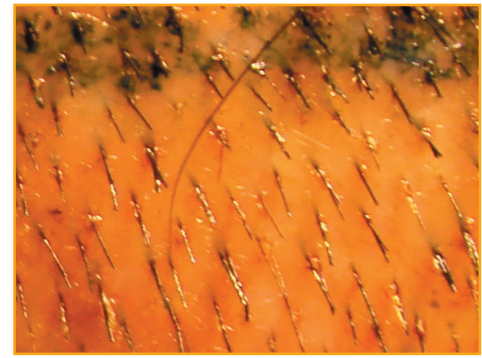

Figure 3. Example of real scalp hair distribution.

be very important and interesting.

Editor's note: Dr. Chang brings up and supports with new data an old topic that has never been resolved: Chubby grafts, according to most studies, appear to grow more hair and, Dr. Chang's data would suggest, larger fiber diameter. Chubby grafts have largely died out, victims to two phenomena. The first is that misuse of the multi-follicular unit graft produces a visibly suboptimal result, whereas the adverse outcome of the single follicular unit graft, a "skinny graft," is invisible; that is, the failure of and/or miniaturized growth is more difficult to recognize. The second reason is marketing and the associated consumer sentiment that rendered the larger graft as "Old School."

Measuring hair fiber diameter is a subject that warrants its own discussion. The micrometer, for instance, has a problem measuring the two axes of the oval shape of most hair. Reasons supporting why the micrometer is still a good tool for this assessment is beyond the scope of this comment. Although the HairCheck is a good substitute in many circumstances, it would be an advancement of our specialty if the single fiber could be measured and accurately correlated with hair mass. - WR

A note from Dr. Michael Beehner: I was fascinated by Dr. Chang's proposition that the final diameter of hairs within a double follicular unit graft is somewhat greater than those in a "single" FU graft. I couldn't tell from Dr. Chang's comments whether his diameter study was done on one patient or more. Obviously, the fewer patients in a study, the greater is the need to corroborate the results with additional patients or studies.

With regard to the observation that the sheer amount of tissue "mass" within a recipient site favors a higher survival rate, I hadn't actually thought of it before in that strict context, but perhaps it is true. Having used multi-follicular unit (MFU) grafts within the central areas of many of my patients for the past 20 years, I have always been impressed with the fact that these grafts always reliably grow out earlier than FU grafts and with apparent full survival just from observation. In the studies I have conducted on survival of the hairs within MFU grafts of 4-6 hairs, the results have usually been very close to $100 \%$. I have always attributed this high rate of survival to the "buffering"

safety provided by the extra tissue within the graft with regard to minimizing trauma in the placing step and also to the fact that hidden telogen hairs are more likely to be included within such grafts and later blossom out and contribute to the number of hairs counted later on. So the fascinating question he raises is the following: Is the mass of the amount of tissue within a recipient site, regardless of whether it is paired or not, more important than the "intactness" of the follicles/grafts within that site?

I agree with Dr. Chang that it would be very interesting to study the "quality" of the hairs grown out from various size grafts, especially with regard to FU grafts that are trimmed either chubby, medium, or skeletonized. In my "chubby vs. skinny" study, which I modeled somewhat after Dr. Seager's similar study, my recollection is that the hairs were almost all relatively "terminal" in appearance, although I certainly did not perform hair diameter measurements.

The one area in which I slightly disagree with Dr. Chang is on the visual effect of 1-hair grafts scattered around as compared with the same number of hairs paired up and arising from half that number of recipient sites. When viewing short, clipped hair stubs as in his photo, it is true that two hairs can almost appear as one and the spaces are very noticeable. But I find that groups of hairs exiting together from the same recipient site, whether it be in pairs of 2 or up to 6, block light much better when the hair grows out than if the same number of hairs was evenly distributed with smaller hair numbers per site. As the hairs grow out, they diverge slightly as they elongate and there is an innate light-blocking quality to such a group of hairs when they emerge together. This is most especially true when it applies to groups of 4 or more hairs.

In closing, I would like to add my conjecture as to why the hair count after a year is sometimes less than one done several months after the study. One possible explanation would be that, as the months pass by, the individual follicles randomize into an asynchronous distribution of those in the anagen phase and those in telogen. Perhaps in the physical tissue climate immediately following a transplant, some follicles are more susceptible to converting to the telogen phase than they would otherwise.

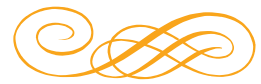

\section{Re: Body dysmorphic disorder}

Greg Williams, FRCS (Plast), Alex Clarke, D. Psych London, UK dr.greg@farjo.com

We read with great interest Dr. Rajput's article titled "Evaluation of body dysmorphic disorder in hair loss patients and benefit after hair restoration" in the July/August 2012 edition of the Forum and your comments in the same edition.

With regards to Dr. Rajput's article, we commend his initiative in exploring this very important area of our practice but would like to make the following comments.

Psychiatrists describe Body Dysmorphic Disorder (BDD) as extreme pre-occupation with a particular feature, in the absence of a severe disfigurement, which has a significant impact on psychological well-being. This is included in DSM-IV as a discrete diagnostic category, with established criteria, often co-morbid with Obsessive Compulsive Disorder (OCD).

Many psychologists take a different conceptual position, suggesting that BDD is the extreme manifestation of appearance related anxiety that marks one end of a continuum with normative discontent at the opposite pole. Some of those with extreme 
appearance anxiety will have a disfiguring condition and others may be concerned with a very minor condition; indeed there is no relationship between severity and psychological distress. In our clinical experience, practitioners in the cosmetic surgery and dermatology settings will see some patients who have BDD (prevalence has been suggested as 1-2\% in the general population but much higher in these settings) but an even greater number of people for whom their appearance fails to meet an ideal and who are strongly motivated to change it. There is certainly evidence of preoccupation and avoidance of certain social activities - but this group is essentially socially anxious or lacking in self-confidence rather than body dysmorphic.

We would suggest that your comments in the co-editors messages reflect your experience largely with this latter group. Indeed, if properly supported, with real understanding (and if necessary modification of) their expectations, very anxious patients commonly report good outcomes as you have suggested. However, those with BDD — the extreme group — are far less likely to respond favourably to surgery. This is the group who undergo multiple procedures without achieving their goals and for whom preoccupation increases rather than reduces over the course of their treatment.

For this reason the UK National Institute for Clinical Excellence (NICE) recommends that patients with BDD be screened out of surgery and referred for psychological assessment. ${ }^{1}$

Whilst all hair transplant practitioners will acknowledge that there is a spectrum of anxiety and fixation in patients who are losing their hair, we maintain that a small subset of these patients will have BDD. Essentially there is no reason why we would not expect to be seeing a similar group of patients across all settings aimed at modifying appearance. It would be beyond the expertise of most hair transplant surgeons to be able to discriminate between those with extreme anxiety/fixation and those with BDD and we would therefore strongly recommend that the experience of a psychologist is sought for all patients who report an excessive preoccupation with their appearance which restricts their ability to live a normal life. They will typically report a strong belief that they are "ugly," seek constant reassurance from other people, and spend excessive time in front of the mirror checking their appearance (see NICE for specific questions). This information is very easy to elicit as part of a clinical assessment.

There is no need to include complex psychometric tools at the screening stage, although for those who are interested in using a standardised measure for clinical or research purposes, we would recommend the COPS cosmetic screening questionnaire ${ }^{2}$ as an alternative to Dr. Rajput's non-standardised measures. A thorough clinical assessment (as above) is enough to highlight concern and trigger onward referral to a psychologist. At this stage the task is one of screening for other problems not making a diagnosis, so being over inclusive in who is referred is not a problem.

Recommending to a patient that they might benefit from seeing a psychologist needs to be done in a sensitive and supportive manner and does open the possibility of the patient seeking an alternative surgeon who might be willing to offer them the surgery they are requesting without this stipulation. However, overanxious patients, including those with or without BDD, often find the input of a psychologist beneficial in identifying the reasons for their excessive concerns, discussing additional strategies for management and setting out very clear goals and expectations of surgery. In the $\mathrm{UK}$, this is now recommended as good practice and helps to frame the provider as offering the highest standard of care.

Establishing a relationship with one or more psychologists who have expertise and experience in this field is essential so that the hair transplant surgeon can confidently directly refer the small number of patients about whom they have concerns. Avoiding operating on patients who do in fact have BDD will save the surgeon a great deal of grief and, in fact, is the appropriate management of the patient. Recommended treatment for BDD is via cognitive behaviour therapy for mild BDD with selective serotonin reuptake inhibitors (SSRIs) for moderate to severe BDD characterised by obsessional features. We therefore respectfully disagree with your suggestion that there is no benefit to labelling someone with BDD and thank you for raising this controversy. We believe further research is required looking at what motivates some patients with hair loss to undergo surgery, how satisfied they are post-operatively and more structured methods to identify those patients who might not be satisfied post-operatively, including those with BDD, prior to offering surgery. We look forward to hearing the views of other readers of the Forum.

\section{References}

1. Obsessive-compulsive disorder: core interventions in the treatment of obsessive-compulsive disorder and body dysmorphic disorder. NICE: 2005. http://www.nice.org.uk/nicemedia/ live/10976/29947/29947.pdf.

2. Veale, D., et al. Development of a cosmetic screening questionnaire (COPS) for screening body dysmorphic disorder. JPRAS. 2012; 65(4):530-532.

Editor's note: I would like to thank Drs. Williams and Clarke for reading, reflecting upon, and responding to my thoughts regarding our patients' emotions concerning their hair loss. (Dr. Williams was gracious in seeking me out in the Bahamas to "apologize" in advance for his differing opinion; none is needed, of course, but what a nice man to be so courteous!) I think our positions are not that far apart. I believe our differences lie in our respectively presumed frequency of BDD. His perception is that "...prevalence has been suggested as $1-2 \%$ in the general population but much higher in... [our hair transplantation surgery] settings." My perception, on the other hand, is that the frequency is at least two or three decimal points to the left of this percentage. If I agreed with his frequency, I would be in complete agreement with his proposals. Actually, I doubt that I could feel fulfilled practicing hair transplantation if it made so many people unhappy. He characterizes this percentage of several percent as patients having a "preoccupation... [regarding their hair that] increases rather than reduces over the course of their treatment." I like this concrete definition as opposed to the others alluded to that deal with abstract levels of anxiety or obsession. Using this definition, I may be seeing my first such patient as I write, although I am still confident that an incomplete sense of satisfaction will be our ultimate outcome. This frequency is literally one in thousands. With this frequency I have trouble losing sleep over my deficiencies in being able to "discriminate between those with extreme anxiety/fixation and those with BDD." I think good documentation and review with the patient of the surgical plan both pre-operatively and post-operatively, good photos, and emphasizing a relationship that is based upon a partnership in striving for what the patient has defined as goals are crucial in keeping the demons of the DSM-IV at bay.

I again thank the doctors for expressing their opinions regarding BDD in the hair loss patient. I am sure their opinions are shared by many readers. As I mentioned in my editorial this month, it is the sharing of ideas that helps us all grow in our mastery of this wonderful specialty. - WR 